ICD-derived Injury Severity Score ICISS, length of stay), nature, and body region were examined.

Results The involvement of motor vehicles was related to more severe injury outcomes for vulnerable road users (between 1.5 and 2.8 times the odds of being serious), as were high speeds (between 1.2 and 1.6 times), alcohol (2.1 times for motorcycles), and helmet non-use (between 1.9 and 2 times). The circumstances of crashes, such as counterpart type and crash location, influenced injury nature and body region.

Conclusions By bringing together circumstance data from police and injury outcome data from hospitals, a greater understanding of the link between crash circumstances and injury outcomes was achieved. Studies using linked data can better inform intervention and treatment for a more holistic approach to the reduction of road trauma for vulnerable road users.

\section{SPEED CONTROL ON MITRAPHAP HIGHWAY WHICH PASSING THROUGH THE MUNICIPALITY OF KHON KAEN PROVINCE BY AUTOMATIC SPEED ENFORCEMENT SYSTEM}

Nittayaporn Srihabua. Khon Kaen Hospital, Thailand

10.1136/injuryprev-2016-042156.864

Background Mitraphap highway is passing through Khon Kaen municipality. The passing length is 14 kilometres. Every year, there are an average of 500 road crashes and at least 15 road accident fatalities occurring on this road. The most common cause of road accidents in this area is related to drivers driving at speeds above the legal speed limit.

Ojectives to reduce vehicle's speed on the 14 kilometres of the Mitraphap highway which passing through Khon Kaen's municipality in order to reduce number of crashes and severity in this high risk area.

Methods Participation Action Research Methodology is used for this project. The research was carried out in 8 steps between January and December 2015. Step 1: Set up a working committee to conduct continuous meetings and implementation. Step 2: Working committee studies high risk areas on Mitraphap highway. Step 3: Use data analysis plan for solutions together. Step 4: Communicate data analysis with key stakeholders. Step 5: Execute prevention measures according to plan by 5.1) setting speed limit to $60 \mathrm{~km} / \mathrm{hr} 5.2$ ) campaign measures to detect driving speed 5.3) Educate on speed control and road traffic injury prevention 5.4) Instal traffic signs and billboard on the road 5.5) instal automatic speed detector cameras ( $24 \mathrm{hr}$.) and charge speed violators. Step 6: Intensive public campaign. Step 7: strict enforcement. Step 8 Analysis for the result of the implementation: The team evaluated effectiveness of the campaign by conducting data analysis and assessed attitudes of drivers toward measures to control driving speed in the controlled areas through face-to-face interviews.

Results $87 \%$ were in favour of enforcing speed limit. $79 \%$ were in agreement of using automatic speed detector cameras. $78 \%$ said they were aware of the speed limit enforced at $60 \mathrm{~km} / \mathrm{hr}$ and $75 \%$ agreed that this is an appropriate speed limit. Prior to enforcing the campaign measures, data revealed that in 2014 there were 559 road accidents with 542 injuries and 22 fatalities. After implementing the campaign measures in 2015, number of road accidents reduced to 355 incidents, road accidents injuries reduced to 296 cases $(\mathrm{OR}=0.24,95 \% \mathrm{CI}:=0.13-0.43)$ and fatalities reduced to 9 cases $(\mathrm{OR}=0.74,95 \% \mathrm{CI}:=0.341 .63)$. Further, in 2015 a total of 4,442 speeding tickets were issued and $69.05 \%$ of violators paid their fines.

Conclusions Results revealed that by using data to plan road accident prevention activities and develop participation of network parties to prevent and solve problems related to road accidents can reduce number of incidents and severity in this high risk area. Further, this activity also strengthened the ability among the network parties and working committee to be able to prevent and solve problems related to road accidents in other high risk areas which should be further implemented on a continuous basis.

\section{BURDEN OF ROAD TRAFFIC INJURIES IN THIKA AND NAIVASHA, KENYA}

${ }^{1}$ Abdulgafoor M Bachani, ${ }^{1}$ Yuen Wai Hung, ${ }^{2}$ Daniel Akunga, ${ }^{3}$ Stephen Mogere,

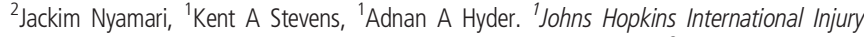
Research Unit, Johns Hopkins Bloomberg School of Public Health; ${ }^{2}$ Kenyatta University; ${ }^{3}$ Roless Institute, Kenya

\subsection{6/injuryprev-2016-042156.865}

Background Road traffic injuries (RTIs) have become one of the leading public health burden in low- and middle-income countries (LMICs). Studies have found RTIs were among the major causes of death and hospital admissions in Kenya. However, most findings were from health care settings and there is a lack of literature on population-based estimates of the burden of road traffic injuries in Kenya.

Methods A cross-sectional household survey was conducted in Thika and Naivasha, Kenya in 2011. Using a cluster random sampling approach, households in the area served by six to eight community health units in the two districts were randomly selected for interview. Upon informed consent, interviewers asked adult respondents about all unintentional injuries to them or members of their households in the past one year, followed by type of injury, and place of occurrence. Additional questions were asked about the road traffic injury or death, if a road traffic injury was reported.

Results The study recruited 669 households in Thika and 439 households in Naivasha, which included a total of 3804 individuals. A small proportion of households approached declined the interview $(2.3 \%$ in Thika, $10.0 \%$ in Naivasha). Unintentional injuries were prevalent in both districts (7.3 in Thika, 5.9\% in Naivasha). RTIs were the top cause of unintentional injuries in Thika (46.8\%), and the second cause of unintentional injuries in Naivasha (27.3\%). Majority of individuals with RTIs sought medical treatment after the injury in Thika (96.5\%), but fewer RTI survivors sought care after RTIs in Naivasha (73.1\%). Reporting of the RTI to police was also low in Naivasha $(66.1 \%$ in Thika vs. $34.6 \%$ in Naivasha).

Conclusions This survey provides a better understanding of the prevalence of RTIs in Thika and Naivasha, the circumstances leading to them, as well as medical care for the injury. This information could be useful in designing and implementing interventions to address this burden. 